\title{
WELL-POSEDNESS AND LARGE DEVIATIONS FOR THE STOCHASTIC PRIMITIVE EQUATIONS IN TWO SPACE DIMENSIONS*
}

\author{
HONGJUN GAO ${ }^{\dagger}$ AND CHENGFENG SUN ${ }^{\ddagger}$
}

\begin{abstract}
The two dimensional primitive equations with multiplicative noise are studied in this paper. The existence and uniqueness of solutions in a fixed probability space and a WentzellFreidlin type large deviation principle for small multiplicative noise by weak convergence method are obtained.
\end{abstract}

Key words. Primitive equation, existence and uniqueness, large deviations.

AMS subject classifications. 35Q99, 60H15, 76M35, 86A05, 86A10.

\section{Introduction}

As a fundamental model in meteorology, the Primitive Equations (PEs) were derived from the Navier-Stokes equations, with rotation, coupled to thermodynamics and salinity diffusion-transport equations, by assuming two important simplifications: the buoyancy forces and stratification effects under the Boussinesq approximation, and the vertical motion by the hydrostatic balance. For further background and detailed physical derivations, we could refer to [9] or [35], for example.

The mathematical study of the PEs originated in a series of articles by J. L. Lions, R. Temam, and S. Wang in the early 1990s [30, 31, 32]. They defined the notions of weak and strong solutions and also proved the existence of weak solutions. Existence of strong solutions (local in time) and their uniqueness were obtain in [22] and [43]. Hu et al. studied the local existence of strong solutions to the primitive equations under the small depth hypothesis in [24]. In [3], Cao and Titi developed a delicate approach to prove that the $L^{6}$-norm of the fluctuation $\widetilde{v}$ of horizontal velocity is bounded, and obtained the global well-posedness for the 3D viscous primitive equations. Another different proof of this result was given by Kobelkov in [27] and [28]. The existence of the attractor was obtained in [25]. In [29], existence and uniqueness for different physically relevant boundary conditions are established with a third method (different from both $[3,27]$ ) that directly treats the pressure terms in the equations. For a general reference on the current research of the (deterministic) mathematical theory for the Primitive equations, we can refer to [39]. Moreover, the deterministic 2D Primitive equations were studied also in [37, 38].

The addition of white noise driven terms to the basic governing equations for a physical system is natural for both practical and theoretical applications. Stochastic solutions of the 2D primitive equations of the ocean and atmosphere with an additive noise has been studied in [14]. Weak and strong random attractors have been ob-

${ }^{*}$ Received: February 3, 2011; accepted (in revised version): August 2, 2011. Communicated by Weinan E.

This work was partially supported by China NSF Grant Nos. 10871097 and 11028102 , National Basic Research Program of China (973 Program) No. 2007CB814800, and Qing Lan Project of Jiangsu Province.

† Jiangsu Key Laboratory for NSLSCS and School of Mathematical Sciences, Nanjing Normal University , Nanjing 210046, China and Center of Nonlinear Science, Nanjing University, Nanjing 210093, China (gaohj@njnu.edu.cn, gaohj@hotmail.com).

$\ddagger$ College of Applied Mathematics, Nanjing University of Finance and Economics, Nanjing, 210046, China (sch200130@163.com). 
tained for the 3D stochastic primitive equations with additive noise in [23] and [18], respectively. The existence and uniqueness of solutions for $2 \mathrm{D}$ stochastic primitive equations with multiplicative noise had been discussed in [21], where they ignored the coupling with the temperature and salinity equations. There have also been other recent works on the stochastic 2D and 3D primitive equations [19, 11], in which a coupling with temperature and salinity equations as well as physically relevant boundary conditions are considered. No small noise conditions are imposed, in contrast with this paper. Recently, a significant literature has developed concerning the NavierStokes equations which are driven by a multiplicative white noise; see [15, 20, 33]. In this paper, we first obtain the well-posedness and general a priori estimates for $2 \mathrm{D}$ stochastic primitive equations with small and more general multiplicative noise which couple the temperature equation by a method different to that in [21]. Our second result is a Wentzell-Freidlin type large deviation principle (LDP) for 2D stochastic primitive equations. There are already several interesting and important papers on LDP and its applications [4]-[8], [12, 13, 17, 26, 36, 41, 42, 44]. Especially in [8], they dealt with a class of abstract nonlinear stochastic models, which covers many 2D hydrodynamical models including 2D Navier-Stokes equations, 2D MHD models, the 2D magnetic Bénard problem and also some shell models of turbulence, but does not include our problem since the mapping $B$ in this paper does not satisfy Condition (C1) of [8]. The idea of our proof by weak convergence [1,2] is similar to [13], so that in this paper we only give the outline of the proof.

This paper is organized as follows. The mathematical formulation for the stochastic primitive equation is in $\S 2$. Then the well-posedness and general a priori estimates for the model are proved in $\S 3$. Finally, a large deviation principle is given in $\S 4$.

\section{Mathematical formulation}

The two dimensional primitive equations can be formally derived from the full three dimensional system under the assumption of invariance with respect to the second horizontal variable $y$ as in [21]. Atmospheric or oceanic motions exhibit fluctuations over a broad range of spatial and temporal scales ranging from centimeters to thousands of kilometers and from seconds to decades and beyond. Such fluctuations can be caused by internal instability processes, as well as by external forcing. As usual, the atmospheric forcing field should be regarded as random; see e.g. [15, 16, 34, 40]. As result, we arrive at the following stochastic evolution system:

$$
\begin{aligned}
\frac{d u^{\varepsilon}}{d t} & =\nu_{1} \Delta u^{\varepsilon}-u^{\varepsilon} \partial_{x} u^{\varepsilon}-w^{\varepsilon} \partial_{z} u^{\varepsilon}-\partial_{x} p+f+\sqrt{\varepsilon} \sigma_{1}\left(t, \phi^{\varepsilon}\right) \dot{W}_{1}, \\
\partial_{z} p & =-\theta, \\
\partial_{x} u^{\varepsilon} & =-\partial_{z} w^{\varepsilon}, \\
\frac{d \theta^{\varepsilon}}{d t} & =\nu_{2} \Delta \theta^{\varepsilon}-u^{\varepsilon} \partial_{x} \theta^{\varepsilon}-w^{\varepsilon} \partial_{z} \theta^{\varepsilon}+q+\sqrt{\varepsilon} \sigma_{2}\left(t, \phi^{\varepsilon}\right) \dot{W}_{2},
\end{aligned}
$$

with velocity $u^{\varepsilon}=u^{\varepsilon}(t, x, z) \in \mathbb{R}$, temperature $\theta^{\varepsilon}=\theta^{\varepsilon}(t, x, z) \in \mathbb{R}, \phi^{\varepsilon}=\left(u^{\varepsilon}, \theta^{\varepsilon}\right)$, pressure $p$, and where $f$ is an external forcing term, $q$ is a given heat source, $(x, z) \in \mathcal{M}=$ $[0, l] \times[-h, 0], t>0$, and $\dot{W}_{1}$ and $\dot{W}_{2}$ are the white noise processes. Here $\Delta$ is the Laplacian operator, and without lost of generality in this paper we take $\nu_{1}, \nu_{2}$ to be 1.

We partition the boundary into the top $\Gamma_{u}=\{z=0\}$, the bottom $\Gamma_{b}=\{z=-h\}$, and the sides $\Gamma_{s}=\{x=0\} \cup\{x=l\}$. In this paper, we consider the following boundary conditions:

$$
\text { on } \Gamma_{u}: \partial_{z} u^{\varepsilon}=0, w^{\varepsilon}=0, \partial_{z} \theta^{\varepsilon}=0,
$$




$$
\begin{aligned}
& \text { on } \Gamma_{b}: \partial_{z} u^{\varepsilon}=0, w^{\varepsilon}=0, \partial_{z} \theta^{\varepsilon}=0, \\
& \text { on } \Gamma_{s}: u^{\varepsilon}=0, \partial_{x} \theta^{\varepsilon}=0 .
\end{aligned}
$$

Due to (2.2) and (2.3), we have that

$$
\begin{aligned}
& w(x, z, t)=-\int_{-h}^{z} \partial_{x} u^{\varepsilon}(x, \xi, t) d \xi \\
& p(x, z, t)=p_{s}(x, t)-\int_{-h}^{z} \theta^{\varepsilon}(x, \xi, t) d \xi
\end{aligned}
$$

Note that $p_{s}$ denotes the surface pressure (with only $x$-dependence). We define the function spaces $H$ and $V$ as follows:

$$
\begin{aligned}
& H=H_{1} \times H_{2}, V=V_{1} \times V_{2}, \\
& H_{1}=\left\{v \in L^{2}(\mathcal{M}) \mid \int_{-h}^{0} v d z=0\right\}, \\
& H_{2}=\left\{\theta \in L^{2}(\mathcal{M})\right\}, \\
& V_{1}=\left\{v \in H^{1}(\mathcal{M})\left|\int_{-h}^{0} v d z=0, v\right|_{\Gamma_{s}}=0\right\}, \\
& V_{2}=\left\{\theta \in H^{1}(\mathcal{M})\right\} .
\end{aligned}
$$

These spaces are endowed with the $L^{2}$ and $H^{1}$ norms, which we respectively denote by $|\cdot|$ and $\|\cdot\|$. The inner products and norms on $V, H$ are given by

$$
\begin{gathered}
\left(U, U_{1}\right)=\left(v, v_{1}\right)+\left(\theta, \theta_{1}\right)=\int_{\mathcal{M}}\left(v v_{1}+\theta \theta_{1}\right) d \mathcal{M}, \\
\left(\left(U, U_{1}\right)\right)=\left(\left(v, v_{1}\right)\right)+\left(\left(\theta, \theta_{1}\right)\right)=\int_{\mathcal{M}}\left(\nabla v \nabla v_{1}+v v_{1}+\nabla \theta \nabla \theta_{1}+\theta \theta_{1}\right) d \mathcal{M}, \\
|U|=(U, U)^{\frac{1}{2}},\|U\|=((U, U))^{\frac{1}{2}},
\end{gathered}
$$

where $U=(v, \theta), U_{1}=\left(v_{1}, \theta_{1}\right) \in V$. We shall also need the intermediate space

$$
\begin{gathered}
Y=Y_{1} \times Y_{2} \\
Y_{1}=\left\{v \in H_{1}, \partial_{z} v \in H_{1}\right\}, Y_{2}=\left\{\theta \in H_{2}, \partial_{z} \theta \in H_{2}\right\} .
\end{gathered}
$$

Let $V^{\prime}$ be the dual space of $V$. We have the dense and continuous embeddings $V \hookrightarrow H=H^{\prime} \hookrightarrow V^{\prime}$ and denote by $\langle\phi, \psi\rangle$ the duality between $\phi \in V$ (resp. $V_{i}$ ) and $\phi \in V^{\prime}\left(\operatorname{resp} . V_{i}^{\prime}\right)$.

Consider an unbounded linear operator $A=\left(A_{1}, A_{2}\right)=(\Delta, \Delta): D(A) \rightarrow H$ with $D(A)=D\left(A_{1}\right) \times D\left(A_{2}\right)$, where

$$
\begin{gathered}
D\left(A_{1}\right)=\left\{u \in V_{1} \cap H^{2}(\mathcal{M}):\left.\partial_{z} u\right|_{\Gamma_{u}}=\left.\partial_{z} u\right|_{\Gamma_{b}}=0\right\} \subset V_{1} \cap H^{2}(\mathcal{M}), \\
D\left(A_{2}\right)=\left\{\theta \in V_{2} \cap H^{2}(\mathcal{M}):\left.\partial_{x} \theta\right|_{\Gamma_{s}}=0,\left.\partial_{z} \theta\right|_{\Gamma_{b}}=\left.\partial_{z} \theta\right|_{\Gamma_{u}}=0\right\} \subset V_{2} \cap H^{2}(\mathcal{M}),
\end{gathered}
$$


and define

$$
\left\langle A_{1} u, v\right\rangle=((u, v)),\left\langle A_{2} \theta, \eta\right\rangle=((\theta, \eta)), \forall u, v \in D\left(A_{1}\right), \forall \theta, \eta \in D\left(A_{2}\right) .
$$

The operator $A_{1}$ is self-adjoint and positive, with compact self-adjoint inverses whose (discrete) eigenvalues are positive. The operator $A_{2}$ is a self-adjoint, nonnegative unbounded operator with eigenvalue 0 and the other (discrete) eigenvalues positive. They map $V_{i}$ to $V_{i}^{\prime}(i=1,2)$.

In accordance with (2.5) we take

$$
\mathcal{W}(v):=-\int_{-h}^{z} \partial_{x} v(x, \widetilde{z}) d \widetilde{z}
$$

and let

$$
B_{1}(u, v):=u \partial_{x} v+\mathcal{W}(u) \partial_{z} v,
$$

where $u \in V_{1}$ and $v \in V_{1}$ or $V_{2}$.

One would like to establish that $B_{1}$ is a well defined and continuous mapping from $V_{1} \times V_{1} \rightarrow V_{1}^{\prime}$ or $V_{1} \times V_{2} \rightarrow V_{2}^{\prime}$ according to

$$
\left\langle B_{1}(u, v), w\right\rangle=b_{1}(u, v, w),
$$

where the associated trilinear form is given by

$$
b_{1}(u, v, w)=\int_{\mathcal{M}}\left(u \partial_{x} v w+\mathcal{W}(u) \partial_{z} v w\right) d \mathcal{M}
$$

In the sequel, when no confusion arises, we denote by $C$ a constant which may change from one line to the next one.

Lemma 2.1 (Estimates for $b_{1}$ and $B_{1}$ ). The trilinear forms $b_{1}$ and $B_{1}$ have the following properties. There exists a constant $C>0$ such that

$$
\begin{aligned}
&\left|b_{1}(u, v, w)\right| \leq C\left(|u|^{\frac{1}{2}}\|u\|^{\frac{1}{2}}\|v\||w|^{\frac{1}{2}}\|w\|^{\frac{1}{2}}+\left|\partial_{x} u\left\|\partial_{z} v\right\| w\right|^{\frac{1}{2}}\|w\|^{\frac{1}{2}}\right), \\
& u \in V_{1}, v \in V_{1}\left(\text { or } V_{2}\right), w \in V_{1}\left(\text { or } V_{2}\right), \\
& b_{1}(u, v, v)=0, \quad u \in V_{1}, v \in V_{1}\left(\text { or } V_{2}\right), \\
&\left\langle B_{1}(u, u), \partial_{z z} u\right\rangle=0, \quad u \in V_{1} .
\end{aligned}
$$

Proof. We only give the proof of (2.17) and (2.19). By Hölder's inequality, Ladyzhenskaya's inequality, we have

$$
\begin{aligned}
\left|b_{1}(u, v, w)\right| \leq & \int_{\mathcal{M}}\left(\left|u \partial_{x} v w\right|+\left|\mathcal{W}(u) \partial_{z} v w\right|\right) d \mathcal{M} \\
\leq & C|u|^{\frac{1}{2}}\|u\|^{\frac{1}{2}}\|v\||w|^{\frac{1}{2}}\|w\|^{\frac{1}{2}}+\int_{0}^{l}\left(\sup _{z \in[-h, 0]}\left\{\int_{-h}^{z} \partial_{x} u d z\right\} \int_{0}^{l}\left|\partial_{z} v w\right| d z\right) d x \\
\leq & \left.C|u|^{\frac{1}{2}}\|u\|^{\frac{1}{2}}\|v\||| w\right|^{\frac{1}{2}}\|w\|^{\frac{1}{2}} \\
& +C \int_{0}^{l}\left(\int_{-h}^{0}\left|\partial_{x} u\right|^{2} d z \cdot \int_{-h}^{0}\left|\partial_{z} v\right|^{2} d z \cdot \int_{-h}^{0}|w|^{2} d z\right)^{1 / 2} d x \\
\leq & \left.C|u|^{\frac{1}{2}}\|u\|^{\frac{1}{2}}\|v\||| w\right|^{\frac{1}{2}}\|w\|^{\frac{1}{2}}
\end{aligned}
$$




$$
\begin{aligned}
&+C \sup _{x \in[0, l]}\left(\int_{-h}^{0}|w|^{2} d z\right)^{1 / 2} \int_{0}^{l}\left(\int_{-h}^{0}\left|\partial_{x} u\right|^{2} d z \cdot \int_{-h}^{0}\left|\partial_{z} v\right|^{2} d z\right)^{1 / 2} d x \\
& \leq C|u|^{\frac{1}{2}}\|u\|^{\frac{1}{2}}\|v\||w|^{\frac{1}{2}}\|w\|^{\frac{1}{2}}+C\left|\partial_{x} u\left\|\partial_{z} v\right\| w\right|^{\frac{1}{2}}\|w\|^{\frac{1}{2}} .
\end{aligned}
$$

Noting (2.15) and (2.16) and regarding boundary conditions, we have

$$
\begin{aligned}
\left\langle B_{1}(u, u), \partial_{z z} u\right\rangle & =\int_{\mathcal{M}}\left(u \partial_{x} u \partial_{z z} u+\mathcal{W}(u) \partial_{z} u \partial_{z z} u\right) d \mathcal{M} \\
& =-\int_{\mathcal{M}}\left[\partial_{z}\left(u \partial_{x} u\right) \partial_{z} u+\partial_{z}\left(\mathcal{W}(u) \partial_{z} u\right) \partial_{z} u\right] d \mathcal{M} \\
& =\int_{\mathcal{M}}\left[-\partial_{x} u\left(\partial_{z} u\right)^{2}-u \partial_{x z} u \partial_{z} u+\frac{1}{2} \partial_{x} u\left(\partial_{z} u\right)^{2}\right] d \mathcal{M} \\
& =0
\end{aligned}
$$

REMARK 2.1. In [19] the authors considered the boundary conditions $\partial_{z} u+\alpha_{u} u=$ $0, \partial_{z} \theta+\alpha_{\theta} \theta=0$ on the top boundary for $u$ and $\theta$, respectively, and defined different function spaces from this paper. Due to the boundary conditions which they considered on the top boundary, in order to deal with the pressure term the authors introduced a projection operator $\mathcal{Q}$ onto $H$ from $L^{2}(\mathcal{M})^{2}$ such that $\mathcal{Q} \partial_{x} p_{s}=0$ and $\left\langle B^{1}(u, u), \partial_{z z} u\right\rangle \neq 0$. Due to the boundary conditions on the top boundary and the definition of $H_{1}$ in this paper, we do not introduce the projection operator, and thus the pressure term remains in equations. Due to the boundary conditions in this paper and since $p_{s}$ depends only on $x$, we have the inner product

$$
\begin{aligned}
\left\langle u, \partial_{x} p_{s}\right\rangle & =\int_{0}^{l} \int_{-h}^{0} u \partial_{x} p_{s} d x d z \\
& =-\int_{0}^{l} p_{s}\left(\partial_{x} \int_{-h}^{0} u d z\right) d x \\
& =0
\end{aligned}
$$

and

$$
\begin{aligned}
\left\langle\partial_{z z} u, \partial_{x} p_{s}\right\rangle & =\int_{0}^{l} \int_{-h}^{0} \partial_{z z} u \partial_{x} p_{s} d x d z \\
& =-\int_{0}^{l} p_{s}\left(\partial_{x} \int_{-h}^{0} \partial_{z z} u d z\right) d x \\
& =-\int_{0}^{l} p_{s}\left(\left.\partial_{x} \partial_{z} u\right|_{z=-h} ^{z=0}\right) d x \\
& =0 .
\end{aligned}
$$

We could now obtain the estimates for $|u|^{2 p}$ and $\left|\partial_{z} u\right|^{2 p}$. If we consider the same boundary conditions as in [19], we should introduce the projection operator $\mathcal{Q}$ and add estimates for $\left\langle B^{1}(u, u), \partial_{z z} u\right\rangle$, for which we could refer to [19].

In the present paper, we assume that $W_{1}(t) \in H_{1}(\mathcal{M}), W_{2}(t) \in H_{2}(\mathcal{M})$ are independent Wiener processes defined on a filtered probability space $\left(\Omega, \mathcal{F}, \mathcal{F}_{t}, \mathbb{P}\right)$, with linear symmetric positive covariant operators $Q_{1}$ and $Q_{2}$, respectively. We denote $Q=\left(Q_{1}, Q_{2}\right)$; this is a linear symmetric positive covariant operator in the Hilbert 
space $H$. We assume that $Q_{1}, Q_{2}$, and thus $Q$ are trace class (and hence compact [10]), i.e., $\operatorname{tr}(Q)<\infty$.

We introduce some additional definitions (see $[42,13])$ in the following.

- Denote by $H_{0}=Q^{\frac{1}{2}} H$ the Hilbert space with scalar product

$$
(\phi, \psi)_{0}=\left(Q^{-\frac{1}{2}} \phi, Q^{-\frac{1}{2}} \psi\right), \forall \phi, \psi \in H_{0},
$$

together with the induced norm $|\cdot|_{0}=\sqrt{(\cdot, \cdot)_{0}}$.

- Let $L_{Q}$ be the space of linear operators $S$ such that $S Q^{\frac{1}{2}}$ is a Hilbert-Schmidt operator (and thus a compact operator [10]) from $H$ to $H$. The norm in the space $L_{Q}$ is defined by $|S|_{L_{Q}}^{2}=\operatorname{tr}\left(S Q S^{*}\right)$, where $S^{*}$ is the adjoint operator of $S$.

- Define $\mathcal{A}$ as the set of $H_{0}$-valued $\left(\mathcal{F}_{t}\right)$-predictable stochastic processes $\phi$ with the property $\int_{0}^{T}|\phi(s)|_{0}^{2} d s<\infty$, a.s. Define

$$
\mathcal{A}_{M}=\left\{\phi \in \mathcal{A}: \phi(\omega) \in S_{M}, \text { a.s. }\right\} .
$$

With this notation, the above primitive equations become

$$
\begin{aligned}
& \frac{d u^{\varepsilon}}{d t}=\Delta u^{\varepsilon}-u^{\varepsilon} \partial_{x} u^{\varepsilon}-\mathcal{W}\left(u^{\varepsilon}\right) \partial_{z} u^{\varepsilon}-\partial_{x} p_{s}+\int_{-h}^{z} \partial_{x} \theta^{\varepsilon}+f+\sqrt{\varepsilon} \sigma_{1}\left(t, \phi^{\varepsilon}\right) \dot{W}_{1}, \\
& \frac{d \theta^{\varepsilon}}{d t}=\Delta \theta^{\varepsilon}-u^{\varepsilon} \partial_{x} \theta^{\varepsilon}-\mathcal{W}\left(u^{\varepsilon}\right) \partial_{z} \theta^{\varepsilon}+q+\sqrt{\varepsilon} \sigma_{2}\left(t, \phi^{\varepsilon}\right) \dot{W}_{2}
\end{aligned}
$$

Thus, we rewrite this system for $\phi^{\varepsilon}=\left(u^{\varepsilon}, \theta^{\varepsilon}\right)$ as

$$
d \phi^{\varepsilon}+\left[A \phi^{\varepsilon}+B\left(\phi^{\varepsilon}, \phi^{\varepsilon}\right)+F\left(\phi^{\varepsilon}\right)\right] d t=R d t+\sqrt{\varepsilon} \sigma\left(t, \phi^{\varepsilon}\right) d W(t),
$$

where $W(t)=\left(W_{1}(t), W_{2}(t)\right)$ and

$$
\begin{aligned}
A \phi^{\varepsilon} & =\left(A_{1} u^{\varepsilon}, A_{2} \theta^{\varepsilon}\right), \\
B\left(\phi^{\varepsilon}\right) & =\left(B_{1}\left(u^{\varepsilon}, u^{\varepsilon}\right), B_{1}\left(u^{\varepsilon}, \theta^{\varepsilon}\right)\right), \\
F \phi^{\varepsilon} & =\left(\partial_{x} p_{s}-\int_{-h}^{z} \partial_{x} \theta^{\varepsilon}, 0\right), \\
R & =(f, q), \\
\sigma\left(t, \phi^{\varepsilon}\right) & =\left(\sigma_{1}\left(t, \phi^{\varepsilon}\right), \sigma_{2}\left(t, \phi^{\varepsilon}\right)\right) .
\end{aligned}
$$

The noise intensity $\sigma:[0, T] \times V \rightarrow L_{Q}\left(H_{0}, H\right)$ is assumed to satisfy the following conditions:

Assumption A: There exist positive constants $K$ and $L$ such that

(A.1) $\sigma \in C\left([0, T] \times H ; L_{Q}\left(H_{0}, H\right)\right)$;

(A.2) $|\sigma(t, \phi)|_{L_{Q}}^{2} \leq K\left(1+\|\phi\|^{2}\right), \quad \forall t \in[0, T], \forall \phi \in V$;

(A.3) $|\sigma(t, \phi)-\sigma(t, \psi)|_{L_{Q}}^{2} \leq L\|\phi-\psi\|^{2}, \quad \forall t \in[0, T], \forall \phi, \psi \in V$.

In order to obtain large deviation, we should introduce the stochastic control equation, let $h \in \mathcal{A}, \varepsilon \geq 0$, and consider the following generalized primitive equations with initial condition $\phi_{h}^{\varepsilon}(0)=\xi$ :

$$
d \phi_{h}^{\varepsilon}(t)+\left[A \phi_{h}^{\varepsilon}(t)+B\left(\phi_{h}^{\varepsilon}(t)\right)+F\left(\phi_{h}^{\varepsilon}\right)\right] d t=R d t+\sqrt{\varepsilon} \sigma\left(\phi_{h}^{\varepsilon}(t)\right) d W(t)+\tilde{\sigma}\left(\phi_{h}^{\varepsilon}(t)\right) h(t) d t .
$$


Then, we introduce another intensity coefficient $\tilde{\sigma} \in C\left([0, T] \times V ; L_{Q}\left(H_{0}, H\right)\right)$ such that

Assumptions $\tilde{\text { A: }}$ There exist positive constants $\tilde{K}$ and $\tilde{L}$ such that

$(\tilde{A} .1) \quad|\tilde{\sigma}(t, \phi)|_{L_{Q}}^{2} \leq \tilde{K}\left(1+|\phi|_{L^{4}}^{2}\right), \quad \forall t \in[0, T], \forall \phi \in L^{4}(D) ;$

$(\tilde{A} .2) \quad|\tilde{\sigma}(t, \phi)-\tilde{\sigma}(t, \psi)|_{L_{Q}}^{2} \leq \tilde{L}|\phi-\psi|_{L^{4}}^{2}, \quad \forall t \in[0, T], \forall \phi, \psi \in L^{4}(D)$.

REMARK 2.2. Continuity condition (A.1) and Lipschitz condition (A.3) imply the growth condition (A.2). Meanwhile, ( $\tilde{A} .2)$ together with the assumption

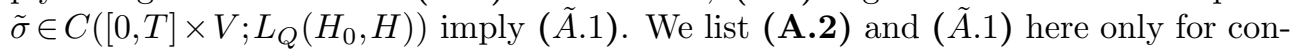
venience.

To obtain Theorem 3.1, we have to give additional assumptions on the $\sigma$ and $\widetilde{\sigma}$. Assumption B: There exists a positive constant $\bar{K}$ such that

(B.1) $\left|\partial_{z} \sigma(t, \phi)\right|_{L_{Q}}^{2} \leq \bar{K}\left(1+\left\|\partial_{z} \phi\right\|^{2}\right), \quad \forall t \in[0, T], \forall \partial_{z} \phi \in V$;

(B.2) $\left|\partial_{z} \tilde{\sigma}(t, \phi)\right|_{L_{Q}}^{2} \leq \bar{K}\left(1+\left\|\partial_{z} \phi\right\|^{2}\right), \quad \forall t \in[0, T], \forall \partial_{z} \phi \in V$.

\section{Well-posedness}

Let $X:=C([0, T] ; H) \cap L^{2}((0, T) ; V)$ denote the Banach space with the norm defined by

$$
\|\phi\|_{X}=\left\{\sup _{0 \leq s \leq T}|\phi(s)|^{2}+\int_{0}^{T}\|\phi(s)\|^{2} d s\right\}^{\frac{1}{2}} .
$$

Recall that an $\left(\mathcal{F}_{t}\right)$-predictable stochastic process $\phi_{h}^{\varepsilon}(t, \omega)$ is called the weak solution for the generalized stochastic primitive problem $(2.29)$ on $[0, T]$, with initial condition $\xi \in X$, if $\phi_{h}^{\varepsilon}$ is in $C([0, T] ; H) \cap L^{2}((0, T) ; V)$, a.s., and satisfies

$$
\begin{aligned}
& \left(\phi_{h}^{\varepsilon}(t), \psi\right)-(\xi, \psi)+\int_{0}^{t}\left[\left\langle\phi_{h}^{\varepsilon}(s), A \psi\right\rangle+\left\langle B\left(\phi_{h}^{\varepsilon}(s)\right), \psi\right\rangle+\left(F\left(\phi_{h}^{\varepsilon}(s)\right), \psi\right)\right] d s \\
= & \int_{0}^{t}(R, \psi) d s+\sqrt{\varepsilon} \int_{0}^{t}\left(\sigma\left(\phi_{h}^{\varepsilon}(s)\right) d W(s) d s, \psi\right)+\int_{0}^{t}\left(\tilde{\sigma}\left(\phi_{h}^{\varepsilon}(s)\right) h(s), \psi\right) d s, \text { a.s. }
\end{aligned}
$$

for all $\psi \in D(A)$ and all $t \in[0, T]$. Note that this solution is a strong one in the probabilistic meaning, that is written in terms of stochastic integrals with respect to the given Brownian motion W. The main result of this section is the following theorem.

Theorem 3.1 (Well-posedness and a priori bounds). Fix $M>0$. Then there exists $\varepsilon_{0}:=\varepsilon_{0}(\bar{K}, K, L, \tilde{K}, \tilde{L}, T, M)>0$ such that the following existence and uniqueness result is true for $0 \leq \varepsilon \leq \varepsilon_{0}$. Let the initial datum $\xi \in Y$ satisfy $\mathbb{E}|\xi|^{4}<\infty, \mathbb{E}\left|\partial_{z} \xi\right|^{4}<\infty$, and let $h \in \mathcal{A}_{M}, f, \partial_{z} f \in L^{4}\left(\Omega ; L^{2}(0, T ; H)\right), q, \partial_{z} q \in L^{4}\left(\Omega ; L^{2}(0, T ; H)\right)$, and $\varepsilon \in\left[0, \varepsilon_{0}\right]$. Then there exists a unique weak solution $\phi_{h}^{\varepsilon}$ of the generalized stochastic primitive problem (2.29) with initial condition $\phi_{h}^{\varepsilon}(0)=\xi \in Y$, such that $\phi_{h}^{\varepsilon} \in X$ a.s. Furthermore, there exists a constant

$$
\begin{array}{r}
C:=C\left(\bar{K}, K, L, \tilde{K}, \tilde{L}, T, M,|f|_{L^{4}\left(\Omega ; L^{2}(0, T ; H)\right)},|q|_{L^{4}\left(\Omega ; L^{2}(0, T ; H)\right)},\right. \\
\left.\left|\partial_{z} f\right|_{L^{4}\left(\Omega ; L^{2}(0, T ; H)\right)},\left|\partial_{z} q\right|_{L^{4}\left(\Omega ; L^{2}(0, T ; H)\right)}\right)
\end{array}
$$

such that for $\varepsilon \in\left[0, \varepsilon_{0}\right]$ and $h \in \mathcal{A}_{M}$,

$$
E\left\|\phi_{h}^{\varepsilon}\right\|_{X}^{2} \leq 1+E\left(\sup _{0 \leq t \leq T}\left|\phi_{h}^{\varepsilon}(t)\right|^{4}+\int_{0}^{T}\left\|\phi_{h}^{\varepsilon}(t)\right\|^{2} d t\right) \leq C\left(1+E|\xi|^{4}\right),
$$


and also

$$
\partial_{z} \phi_{h}^{\varepsilon}(t) \in L^{4}\left(\Omega, L^{\infty}(0, T ; H) \cap L^{2}(0, T ; V)\right)
$$

For $\phi=(u, \theta) \in V$, define

$$
E(\phi)=-A \phi-B(\phi)-F(\phi)+R .
$$

We first obtain monotonicity properties of $E$.

Lemma 3.2. Assume that $\phi=(u, \theta) \in V$ and $\psi=(v, \eta) \in V$. We have

$$
\langle E(\phi)-E(\psi), \phi-\psi\rangle+\frac{1}{2}\|\phi-\psi\|^{2} \leq C|\phi-\psi|\|\phi-\psi\|\|\psi\|+C\left(1+\left|\partial_{z} \psi\right|^{4}\right)|\phi-\psi|^{2} .
$$

Proof. Set $U=u-v, \Theta=\theta-\eta$ and $\Phi=\phi-\psi:=(U, \Theta)$. We deduce

$$
\begin{aligned}
\langle E(\phi)-E(\psi), \Phi\rangle & =-\langle A(\phi)-A(\psi), \Phi\rangle-\langle B(\phi)-B(\psi), \Phi\rangle-\langle F(\phi)-F(\psi), \Phi\rangle \\
& \equiv I_{1}+I_{2}+I_{3} .
\end{aligned}
$$

Integrating by parts, using Lemma (2.1), Hölder's inequality, Ladyzhenskaya's inequality for two-dimensional domain, and Young's inequality, we have

$$
\begin{gathered}
I_{1}=-|\nabla(u-v)|^{2}-|\nabla(\theta-\eta)|^{2}=-\|\phi-\psi\|^{2} \\
I_{2}=b_{1}(u-v, u-v, v)+b_{1}(u-v, \theta-\eta, \eta) \\
\leq|u-v|_{L^{4}}\|v\||u-v|_{L^{4}}+|u-v|_{L^{4}}\|\eta\||\theta-\eta|_{L^{4}} \\
+\left|\partial_{x}(u-v)\left\|\partial_{z} v\right\| u-v\right|^{\frac{1}{2}}\|u-v\|^{\frac{1}{2}}+\left|\partial_{x}(u-v)\left\|\partial_{z} \eta\right\| \theta-\eta\right|^{\frac{1}{2}}\|\theta-\eta\|^{\frac{1}{2}} \\
\leq C|u-v|\|u-v\|\|v\|+C|u-v|^{\frac{1}{2}}\|u-v\|^{\frac{1}{2}}\|\eta\||\theta-\eta|^{\frac{1}{2}}\|\theta-\eta\|^{\frac{1}{2}} \\
+\|u-v\|^{\frac{3}{2}}\left|\partial_{z} v\left\|u-\left.v\right|^{\frac{1}{2}}+\right\| u-v\left\|^{\frac{1}{2}}\left|\|\theta-\eta\|^{\frac{1}{2}}\right| \partial_{z} \eta\right\| \theta-\eta\right|^{\frac{1}{2}} \\
\leq C|\phi-\psi|\|\phi-\psi\|(\|v\|+\|\eta\|)+C\|\phi-\psi\|^{\frac{3}{2}}|\phi-\psi|^{\frac{1}{2}}\left|\partial_{z} \psi\right| \\
\leq C|\phi-\psi|\|\phi-\psi\|\|\psi\|+\frac{1}{4}\|\phi-\psi\|^{2}+C\left|\partial_{z} \psi\right|^{4}|\phi-\psi|^{2} \\
I_{3}=\int_{M}\left(\int_{-h}^{z} \partial_{x}(\theta-\eta) d \widetilde{z} \cdot(u-v)\right) d M \\
\leq \int_{M}\left(\int_{-h}^{0}\left|\partial_{x}(\theta-\eta)\right| d \widetilde{z} \cdot|u-v|\right) d M \\
\leq h\left|\partial _ { x } ( \theta - \eta ) \left\|u-v\left|\leq \frac{1}{4}\|\phi-\psi\|^{2}+C\right| \phi-\left.\psi\right|^{2} .\right.\right.
\end{gathered}
$$

Combining $I_{1}, I_{2}$, and $I_{3}$, we end the proof.

We now introduce the Galerkin systems associated to the original equation and establish some uniform a priori estimates. For any $n \geq 1$, let $H_{n}=\operatorname{span}\left(e_{1}, \cdots, e_{n}\right) \subset$ $\operatorname{Dom}(A)$ and let $P_{n}: H \rightarrow H_{n}$ denote the orthogonal projection onto $H_{n}$. Note that 
$P_{n}$ contracts the $H$ and $V$ norms. Suppose that the $H$-valued Wiener process $W$ with covariance operator $Q$ is such that

$$
P_{n} Q^{\frac{1}{2}}=Q^{\frac{1}{2}} P_{n}, n \geq 1,
$$

which is true if $Q h=\sum_{n \geq 1} \lambda_{n} e_{n}$ with trace $\sum_{n \geq 1} \lambda_{n}<\infty$. Then for $H_{0}=Q^{\frac{1}{2}} H$ and $(\phi, \psi)_{0}=\left(Q^{-\frac{1}{2}} \phi, Q^{-\frac{1}{2}} \psi\right)$, for $\phi, \psi \in H_{0}$, we see that $P_{n}: H_{0} \rightarrow H_{0} \cap H_{n}$ contracts the $H$ and $H_{0}$ norms. Let $W_{n}=P_{n} W, \sigma_{n}=P_{n} \sigma$ and $\tilde{\sigma}_{n}=P_{n} \tilde{\sigma}$.

For $h \in \mathcal{A}_{M}$, we consider the stochastic ordinary differential equation on the $n$ dimensional space $H_{n}$ defined by

$$
d\left(\phi_{n, h}^{\varepsilon}, \psi\right)=\left[\left\langle E\left(\phi_{n, h}^{\varepsilon}\right), \psi\right\rangle+\left(\tilde{\sigma}_{n}\left(\phi_{n, h}^{\varepsilon}\right) h, \psi\right)\right] d t+\sqrt{\varepsilon}\left(\sigma_{n}\left(\phi_{n, h}^{\varepsilon}\right) d W_{n}, \psi\right),
$$

for $\psi=(v, \eta) \in H_{n}$ and $\phi_{n, h}^{\varepsilon}(0)=P_{n} \xi$.

We note that the formulation (3.7) allows one to treat $\phi_{n, h}^{\varepsilon}$ as a process in $R^{n}$. Hence, by a well-posedness result for stochastic ordinary differential equations, there exists a maximal solution to (3.7), i.e., a stopping time $\tau_{n, h}^{\varepsilon} \leq T$ such that (3.7) holds for $t<\tau_{n, h}^{\varepsilon}$ and as $t \uparrow \tau_{n, h}^{\varepsilon}<T,\left|\phi_{n, h}^{\varepsilon}(t)\right| \rightarrow \infty$. One can then apply the finite dimensional Itô's calculus to the above Galerkin systems. We next establish some uniform estimates on $\phi_{n, h}^{\varepsilon}$ (independent of $\mathrm{n}$ ). For every $N>0$, set

$$
\tau_{N}=\inf \left\{t:\left|\phi_{n, h}^{\varepsilon}(t)\right| \geq N\right\} \wedge \inf \left\{t:\left|\partial_{z} \phi_{n, h}^{\varepsilon}(t)\right| \geq N\right\} \wedge T .
$$

The following proposition provides the (global) existence and uniqueness of approximate solutions and also their uniform (a priori) estimates. This is the main preliminary step in the proof of Theorem 3.1.

Proposition 3.1. There exists $\varepsilon_{0, p}:=\varepsilon_{0, p}(K, \tilde{K}, T, M)$ such that for $0 \leq \varepsilon \leq \varepsilon_{0, p}$ the following result holds for an integer $p \geq 1$ (with the convention $x^{0}=1$ ). Let $h \in \mathcal{A}_{M}$, $f, q \in L^{2 p}\left(\Omega ; L^{2}(0, T ; H)\right)$, and $\xi \in L^{2 p}(\Omega, H)$. Then the equation (3.7) has a unique solution with a modification $\phi_{n, h}^{\varepsilon} \in C\left([0, T], H_{n}\right)$ satisfying

$$
\begin{aligned}
& \sup _{n} \mathbb{E}\left(\sup _{0 \leq t \leq T}\left|\phi_{n, h}^{\varepsilon}(t)\right|^{2 p}+\int_{0}^{T}\left\|\phi_{n, h}^{\varepsilon}(s)\right\|^{2}\left|\phi_{n, h}^{\varepsilon}(s)\right|^{2(p-1)} d s\right) \\
& \leq C\left(p, K, \tilde{K}, T, M,|f|_{L^{2 p}\left(\Omega ; L^{2}(0, T ; H)\right)},|q|_{L^{2 p}\left(\Omega ; L^{2}(0, T ; H)\right)}\right)\left(\mathbb{E}|\xi|^{2 p}+1\right) .
\end{aligned}
$$

Proof. Itô's formula yields that for $t \in[0, T]$ and $\tau_{N}$ defined by (3.8),

$$
\begin{aligned}
\left|\phi_{n, h}^{\varepsilon}\left(t \wedge \tau_{N}\right)\right|^{2}=\left|P_{n} \xi\right|^{2} & +2 \sqrt{\varepsilon} \int_{0}^{t \wedge \tau_{N}}\left(\sigma_{n}\left(\phi_{n, h}^{\varepsilon}(s)\right) d W_{n}(s), \phi_{n, h}^{\varepsilon}(s)\right) d s \\
& +2 \int_{0}^{t \wedge \tau_{N}}\left\langle E\left(\phi_{n, h}^{\varepsilon}(s)\right), \phi_{n, h}^{\varepsilon}(s)\right\rangle d s \\
& +2 \int_{0}^{t \wedge \tau_{N}}\left(\tilde{\sigma}_{n}\left(\phi_{n, h}^{\varepsilon}(s)\right) h(s), \phi_{n, h}^{\varepsilon}(s)\right) d s \\
& +\varepsilon \int_{0}^{t \wedge \tau_{N}}\left|\sigma_{n}\left(\phi_{n, h}^{\varepsilon}(s)\right) P_{n}\right|_{L_{Q}}^{2} d s .
\end{aligned}
$$

Applying again Itô's formula for $x^{p}$ when $p \geq 2$, using Lemma 2.1, and with the convention $p(p-1) x^{p-2}=0$ for $p=1$, this yields for $t \in[0, T]$,

$$
\left|\phi_{n, h}^{\varepsilon}\left(t \wedge \tau_{N}\right)\right|^{2 p}+2 p \int_{0}^{t \wedge \tau_{N}}\left|\phi_{n, h}^{\varepsilon}(r)\right|^{2(p-1)}\left[\left\|u_{n, h}^{\varepsilon}(r)\right\|^{2}+\left\|\theta_{n, h}^{\varepsilon}(r)\right\|^{2}\right] d r
$$


584

$$
\leq\left|P_{n} \xi\right|^{2 p}+\sum_{1 \leq j \leq 6} T_{j}(t)
$$

where

$$
\begin{aligned}
& T_{1}(t)=2 p \int_{0}^{t \wedge \tau_{N}}\left|\left\langle\phi_{n, h}^{\varepsilon}, F\left(\phi_{n, h}^{\varepsilon}\right)\right\rangle\right|\left|\phi_{n, h}^{\varepsilon}(r)\right|^{2(p-1)} d r \\
& T_{2}(t)=2 p \int_{0}^{t \wedge \tau_{N}}\left|\left\langle\phi_{n, h}^{\varepsilon}, R\right\rangle\right|\left|\phi_{n, h}^{\varepsilon}(r)\right|^{2(p-1)} d r \\
& T_{3}(t)=\left.2 p \sqrt{\varepsilon}\left|\int_{0}^{t \wedge \tau_{N}}\left(\sigma_{n}\left(\phi_{n, h}^{\varepsilon}(r)\right) d W_{n}(r), \phi_{n, h}^{\varepsilon}(r)\right)\right| \phi_{n, h}^{\varepsilon}(r)\right|^{2(p-1)} d r \mid, \\
& T_{4}(t)=2 p \int_{0}^{t \wedge \tau_{N}}\left|\left(\tilde{\sigma}_{n}\left(\phi_{n, h}^{\varepsilon}(r)\right) h(r), \phi_{n, h}^{\varepsilon}(r)\right)\right|\left|\phi_{n, h}^{\varepsilon}(r)\right|^{2(p-1)} d r \\
& T_{5}(t)=p \varepsilon \int_{0}^{t \wedge \tau_{N}}\left|\sigma_{n}\left(\phi_{n, h}^{\varepsilon}(r)\right) P_{n}\right|_{L_{Q}}^{2}\left|\phi_{n, h}^{\varepsilon}(r)\right|^{2(p-1)} d r, \\
& T_{6}(t)=2 p(p-1) \varepsilon \int_{0}^{t \wedge \tau_{N}}\left|\Pi_{n} \sigma_{n}^{*}\left(\phi_{n, h}^{\varepsilon}(r)\right) \phi_{n, h}^{\varepsilon}(r)\right|_{H_{0}}^{2}\left|\phi_{n, h}^{\varepsilon}(r)\right|^{2(p-2)} d r .
\end{aligned}
$$

Hölder's inequality and Young's inequality imply that

$$
\begin{aligned}
T_{1}(t) & \leq 2 p \int_{0}^{t \wedge \tau_{N}}\left|\phi_{n, h}^{\varepsilon}(r)\right|^{2(p-1)} \int_{M}\left(\left(\int_{-h}^{0}\left|\partial_{x} \theta_{n, h}^{\varepsilon}(r)\right| d z\right) \cdot\left|u_{n, h}^{\varepsilon}(r)\right|\right) d M d r \\
& \leq 2 p \int_{0}^{t \wedge \tau_{N}}\left|\phi_{n, h}^{\varepsilon}(r)\right|^{2(p-1)}\left[h\left|\partial_{x} \theta_{n, h}^{\varepsilon}(r)\right|\left|u_{n, h}^{\varepsilon}(r)\right|\right] d r \\
& \leq \frac{1}{12} \int_{0}^{t \wedge \tau_{N}}\left|\phi_{n, h}^{\varepsilon}(r)\right|^{2(p-1)}\left\|\phi_{n, h}^{\varepsilon}(r)\right\|^{2} d r+C_{1} \int_{0}^{t \wedge \tau_{N}}\left|\phi_{n, h}^{\varepsilon}(r)\right|^{2 p} d r
\end{aligned}
$$

and

$$
\begin{aligned}
T_{2}(t)= & 2 p \int_{0}^{t \wedge \tau_{N}}\left[\left(u_{n, h}^{\varepsilon}(r), f\right)+\left(\theta_{n, h}^{\varepsilon}(r), q\right)\right]\left|\phi_{n, h}^{\varepsilon}(r)\right|^{2(p-1)} d r \\
\leq & \frac{1}{12} \int_{0}^{t \wedge \tau_{N}}\left|\phi_{n, h}^{\varepsilon}(r)\right|^{2(p-1)}\left\|\phi_{n, h}^{\varepsilon}(r)\right\|^{2} d r \\
& +C_{2} \sup _{0 \leq s \leq t \wedge \tau_{N}}\left|\phi_{n, h}^{\varepsilon}(r)\right|^{2(p-1)} \int_{0}^{t \wedge \tau_{N}}\left(|f(r)|^{2}+|q(r)|^{2}\right) d r \\
\leq & \frac{1}{12} \int_{0}^{t \wedge \tau_{N}}\left|\phi_{n, h}^{\varepsilon}(r)\right|^{2(p-1)}\left\|\phi_{n, h}^{\varepsilon}(r)\right\|^{2} d r \\
& +\frac{1}{2} \sup _{0 \leq s \leq t \wedge \tau_{N}}\left|\phi_{n, h}^{\varepsilon}\right|^{2 p}+C_{2}\left(|f|_{L^{2}\left(0, t \wedge \tau_{N} ; H\right)}^{2 p}+|q|_{L^{2}\left(0, t \wedge \tau_{N} ; H\right)}^{2 p}\right) .
\end{aligned}
$$

Using the Cauchy-Schwarz inequality and ( $\widetilde{A} .1)$, we get

$$
\begin{aligned}
T_{4}(t) \leq & 2 p \int_{0}^{t \wedge \tau_{N}}\left[\tilde{K}\left(1+\left\|\phi_{n, h}^{\varepsilon}(r)\right\|^{2}\right)\right]^{\frac{1}{2}}|h(r)|_{0}\left|\phi_{n, h}^{\varepsilon}(r)\right|^{2 p-1} d r \\
\leq & \frac{1}{12} \int_{0}^{t \wedge \tau_{N}}\left\|\phi_{n, h}^{\varepsilon}(r)\right\|^{2}\left|\phi_{n, h}^{\varepsilon}(r)\right|^{2(p-1)} d r+C_{4} \int_{0}^{t \wedge \tau_{N}}|h(r)|_{0}^{2}\left|\phi_{n, h}^{\varepsilon}(r)\right|^{2 p} d r \\
& +\frac{1}{12} \int_{0}^{t \wedge \tau_{N}}\left|\phi_{n, h}^{\varepsilon}(r)\right|^{2(p-1)} d r
\end{aligned}
$$


Using (A.2), we deduce that

$$
\begin{aligned}
T_{5}(t)+T_{6}(t) \leq & 2 p^{2} K \varepsilon \int_{0}^{t \wedge \tau_{N}}\left\|\phi_{n, h}^{\varepsilon}(r)\right\|^{2}\left|\phi_{n, h}^{\varepsilon}(r)\right|^{2(p-1)} d r \\
& +2 p^{2} K \varepsilon \int_{0}^{t \wedge \tau_{N}}\left|\phi_{n, h}^{\varepsilon}(r)\right|^{2(p-1)} d r
\end{aligned}
$$

Finally, the Burkholder-Davies-Gundy inequality (see [10]), (A.2), and Schwarz's inequality yield that for $t \in[0, T]$ and $\delta_{3}>0$,

$$
\begin{aligned}
\mathbb{E}\left(\sup _{0 \leq s \leq t}\left|T_{3}(s)\right|\right) \leq & 6 p \sqrt{\varepsilon} \mathbb{E}\left\{\int_{0}^{t \wedge \tau_{N}}\left|\phi_{n, h}^{\varepsilon}(r)\right|^{2(2 p-1)}\left|\sigma_{n, h}\left(\phi_{n, h}^{\varepsilon}(r)\right) P_{n}\right|_{L_{Q}}^{2} d r\right\}^{\frac{1}{2}} \\
\leq & \delta_{3} \mathbb{E}\left(\sup _{0 \leq s \leq t \wedge \tau_{N}}\left|\phi_{n, h}^{\varepsilon}(s)\right|^{2 p}\right)+\frac{9 p^{2} K \varepsilon}{\delta_{3}} \mathbb{E} \int_{0}^{t \wedge \tau_{N}}\left|\phi_{n, h}^{\varepsilon}(r)\right|^{2(p-1)} d r \\
& +\frac{9 p^{2} K \varepsilon}{\delta_{3}} \mathbb{E} \int_{0}^{t \wedge \tau_{N}}\left\|\phi_{n, h}^{\varepsilon}(r)\right\|^{2}\left|\phi_{n, h}^{\varepsilon}(r)\right|^{2(p-1)} d r
\end{aligned}
$$

Consider the following property $I(i)$ for an integer $i \geq 0$ :

I(i) There exists $\varepsilon_{0, i}:=\varepsilon_{0, i}(K, \tilde{K}, T, M)>0$ such that for $0 \leq \varepsilon \leq \varepsilon_{0, i}$,

$$
\sup _{n} \mathbb{E} \int_{0}^{t \wedge \tau_{N}}\left|\phi_{n, h}^{\varepsilon}(r)\right|^{2 i} d r \leq C(i):=C(i, K, \tilde{K}, T, M)<+\infty .
$$

The property $I(0)$ obviously holds with $\varepsilon_{0,0}=1$ and $C(0)=T$. Assume that for some integer $i$ with $1 \leq i \leq p$, the property $\mathrm{I}(\mathrm{i}-1)$ holds; we prove that $\mathrm{I}(\mathrm{i})$ holds. Here we mainly use a version of Gronwall's lemma $[8,12,13]$.

By setting

$$
\begin{aligned}
\varphi_{i}(r)= & 2\left(C_{1}+C_{4}|h(r)|_{0}^{2}\right), \\
Z= & 2\left(\frac{1}{12}+2 i^{2} K \varepsilon\right) \int_{0}^{\tau_{N}}\left|\phi_{n, h}^{\varepsilon}(r)\right|^{2(i-1)} d r+2|\xi|^{2 i} \\
& +2 C_{2}\left(|f|_{L^{2}(0, T ; H)}^{2 i}+|q|_{L^{2}(0, T ; H)}^{2 i}\right), \\
X(t)= & \sup _{0 \leq s \leq t}\left|\phi_{n, h}^{\varepsilon}\left(s \wedge \tau_{N}\right)\right|^{2 i}, \\
Y(t)= & \int_{0}^{t \wedge \tau_{N}}\left\|\phi_{n, h}^{\varepsilon}(s)\right\|^{2}\left|\phi_{n, h}^{\varepsilon}(s)\right|^{2(i-1)} d s, \\
I(t)= & \left.\sup _{0 \leq s \leq t}\left|2 i \sqrt{\varepsilon} \int_{0}^{t \wedge \tau_{N}}\left(\sigma_{n}\left(\phi_{n, h}^{\varepsilon}(r)\right) d W_{n}(r), \phi_{n, h}^{\varepsilon}(r)\right)\right| \phi_{n, h}^{\varepsilon}(r)\right|^{2(i-1)} \mid,
\end{aligned}
$$

we have $\int_{0}^{T} \varphi_{i}(s) d s \leq C_{i}(M):=2 C_{1} T+C_{4} M$. Let $\alpha=2\left(2 p-\frac{1}{4}-2 p^{2} K \varepsilon\right), \quad \beta=\delta_{3}=$ $\frac{1}{2\left[1+C_{i}(M) e^{C_{i}(M)}\right]}$, and $\tilde{C}=\frac{9 i^{2} K}{\delta_{3}} \mathbb{E} \int_{0}^{\tau_{N}}\left|\phi_{n, h}^{\varepsilon}(s)\right|^{2(i-1)} d s$. If we choose $\varepsilon$ small enough to satisfy $\varepsilon \leq \frac{2 \delta_{3}^{2}\left(2 i-\frac{1}{4}\right)}{9 i^{2} K+4 i^{2} K \delta_{3}^{2}}$, then we have $\gamma=\frac{9 p^{2} K \varepsilon}{\delta_{3}} \leq \alpha \beta$. Lastly, letting $\varepsilon_{0, i}=$ $\frac{2 \delta_{3}^{2}\left(2 i-\frac{1}{4}\right)}{9 i^{2} K+4 i^{2} K \delta_{3}^{2}} \wedge \varepsilon_{0, i-1}$, and using Gronwall's lemma [8, 12, 13], we obtain $\mathbf{I}(\mathbf{i})$.

An induction argument shows that $I(p-1)$ holds, and hence the previous computations with $i=p$ yield that for $t=T$ and $0 \leq \varepsilon \leq \varepsilon_{0, p}$,

$$
\sup _{n} \mathbb{E}\left(\sup _{0 \leq s \leq \tau_{N}}\left|\phi_{n, h}^{\varepsilon}(s)\right|^{2 p}+\int_{0}^{\tau_{N}}\left\|\phi_{n, h}^{\varepsilon}(s)\right\|^{2}\left|\phi_{n, h}^{\varepsilon}(s)\right|^{2(p-1)} d s\right) \leq C(p, K, \tilde{K}, T, M) .
$$


By the definition of $\tau_{n, h}$ and (3.8), we know $\sup _{0<s<t \wedge \tau_{N}}\left|\phi_{n, h}(s)\right| \rightarrow \infty$, and $\tau_{N} \uparrow \tau_{n, h}$ on $\left\{\tau_{n, h}<T\right\}$, as $N \rightarrow \infty$. Hence, by the above estimate we have $\mathbb{P}\left(\tau_{n, h}<T\right)=0$ and for almost all $\omega$, for $N(\omega)$ large enough, $\tau_{N(\omega)}(\omega)=T$. Thus we complete the proof of the proposition.

Proposition 3.2. There exists $\varepsilon_{0, p}:=\varepsilon_{0, p}(K, \tilde{K}, \bar{K}, T, M)$ such that for $0 \leq \varepsilon \leq \varepsilon_{0, p}$ the following result holds: Let $h \in \mathcal{A}_{M}, \partial_{z} f, \partial_{z} q \in L^{2 p}\left(\Omega ; L^{2}(0, T ; H)\right)$, and $\partial_{z} \xi \in$ $L^{2 p}(\Omega, H)$. Then we have

$$
\begin{aligned}
\sup _{n} \mathbb{E} & \left(\sup _{0 \leq t \leq T}\left|\partial_{z} \phi_{n, h}^{\varepsilon}(t)\right|^{2 p}+\int_{0}^{T}\left\|\partial_{z} \phi_{n, h}^{\varepsilon}(s)\right\|^{2}\left|\partial_{z} \phi_{n, h}^{\varepsilon}(s)\right|^{2(p-1)} d s\right) \\
& \leq C\left(K, \tilde{K}, \bar{K}, T, M,\left|\partial_{z} f\right|_{L^{2 p}\left(\Omega ; L^{2}(0, T ; H)\right)},\left|\partial_{z} q\right|_{L^{2 p}\left(\Omega ; L^{2}(0, T ; H)\right)}\right)\left(\mathbb{E}\left|\partial_{z} \xi\right|^{2 p}+1\right) .
\end{aligned}
$$

Proof. Applying Itô's formula for $\left|\partial_{z} \phi_{n, h}^{\varepsilon}\right|^{2 p}$, for $t \in[0, T]$ and $\tau_{N}$ defined by (3.8), we get

$$
\begin{aligned}
& \left|\partial_{z} \phi_{n, h}^{\varepsilon}\left(t \wedge \tau_{N}\right)\right|^{2 p}+2 p \int_{0}^{t \wedge \tau_{N}}\left|\partial_{z} \phi_{n, h}^{\varepsilon}(r)\right|^{2(p-1)}\left[\left\|\partial_{z} u_{n, h}^{\varepsilon}(r)\right\|^{2}+\left\|\partial_{z} \theta_{n, h}^{\varepsilon}(r)\right\|^{2}\right] d r \\
\leq & \left|P_{n} \partial_{z} \xi\right|^{2 p}+\sum_{1 \leq j \leq 6} J_{j}(t)
\end{aligned}
$$

where

$$
\begin{aligned}
& J_{1}(t)=2 p \int_{0}^{t \wedge \tau_{N}}\left|\left\langle\partial_{z z} \phi_{n, h}^{\varepsilon}, B\left(\phi_{n, h}^{\varepsilon}\right)\right\rangle\right|\left|\partial_{z} \phi_{n, h}^{\varepsilon}(r)\right|^{2(p-1)} d r \\
& J_{2}(t)=2 p \int_{0}^{t \wedge \tau_{N}}\left|\left\langle\partial_{z z} \phi_{n, h}^{\varepsilon}, F\left(\phi_{n, h}^{\varepsilon}\right)\right\rangle\right|\left|\partial_{z} \phi_{n, h}^{\varepsilon}(r)\right|^{2(p-1)} d r \\
& J_{3}(t)=2 p \int_{0}^{t \wedge \tau_{N}}\left|\left\langle\partial_{z z} \phi_{n, h}^{\varepsilon}, R\right\rangle\right|\left|\partial_{z} \phi_{n, h}^{\varepsilon}(r)\right|^{2(p-1)} d r \\
& J_{4}(t)=\left.2 p \sqrt{\varepsilon}\left|\int_{0}^{t \wedge \tau_{N}}\left(\sigma_{n}\left(\phi_{n, h}^{\varepsilon}(r)\right) d W_{n}(r), \partial_{z z} \phi_{n, h}^{\varepsilon}(r)\right)\right| \partial_{z} \phi_{n, h}^{\varepsilon}(r)\right|^{2(p-1)} d r \mid \\
& J_{5}(t)=2 p \int_{0}^{t \wedge \tau_{N}}\left|\left(\tilde{\sigma}_{n}\left(\phi_{n, h}^{\varepsilon}(r)\right) h(r), \partial_{z z} \phi_{n, h}^{\varepsilon}(r)\right)\right|\left|\partial_{z} \phi_{n, h}^{\varepsilon}(r)\right|^{2(p-1)} d r \\
& J_{6}(t)=p \varepsilon \int_{0}^{t \wedge \tau_{N}}\left|\partial_{z} \sigma_{n}\left(\phi_{n, h}^{\varepsilon}(r)\right) P_{n}\right|_{L_{Q}}^{2}\left|\partial_{z} \phi_{n, h}^{\varepsilon}(r)\right|^{2(p-1)} d r \\
& J_{7}(t)=2 p(p-1) \varepsilon \int_{0}^{t \wedge \tau_{N}}\left|\Pi_{n}\left(\partial_{z} \sigma_{n}\right)^{*}\left(\phi_{n, h}^{\varepsilon}(r)\right) \partial_{z} \phi_{n, h}^{\varepsilon}(r)\right|_{H_{0}}^{2}\left|\partial_{z} \phi_{n, h}^{\varepsilon}(r)\right|^{2(p-2)} d r .
\end{aligned}
$$

Note that by (2.19), we have

$$
J_{1}(t)=2 p \int_{0}^{t \wedge \tau_{N}}\left|\partial_{z} \phi_{n, h}^{\varepsilon}(r)\right|^{2(p-1)}\left|b_{1}\left(u_{n, h}^{\varepsilon}(r), \theta_{n, h}^{\varepsilon}(r), \partial_{z z} \theta_{n, h}^{\varepsilon}(r)\right)\right| d r .
$$

Integrating by parts and using Hölder's inequality, Ladyzhenskaya's inequality, and Young's inequality, one infers that

$$
J_{1}(t) \leq 2 p \int_{0}^{t \wedge \tau_{N}}\left|\partial_{z} \phi_{n, h}^{\varepsilon}(r)\right|^{2(p-1)} \int_{\mathcal{M}}\left|\partial_{z} \theta_{n, h}^{\varepsilon}(r)\left\|\partial_{z} u_{n, h}^{\varepsilon}(r)\right\| \partial_{x} \theta_{n, h}^{\varepsilon}(r)\right| d \mathcal{M} d r
$$




$$
\begin{aligned}
& +2 p \int_{0}^{t \wedge \tau_{N}}\left|\partial_{z} \phi_{n, h}^{\varepsilon}(r)\right|^{2(p-1)} \int_{\mathcal{M}}\left|\partial_{z} \theta_{n, h}^{\varepsilon}(r)\right|^{2}\left|\partial_{x} u_{n, h}^{\varepsilon}(r)\right| d \mathcal{M} d r \\
\leq & 4 p \int_{0}^{t \wedge \tau_{N}}\left|\partial_{z} \phi_{n, h}^{\varepsilon}(r)\right|^{2(p-1)}\left|\partial_{z} \phi_{n, h}^{\varepsilon}(r)\right|_{L^{4}}^{2}\left|\partial_{x} \phi_{n, h}^{\varepsilon}(r)\right| d r \\
\leq & \frac{1}{12} \int_{0}^{t \wedge \tau_{N}}\left|\partial_{z} \phi_{n, h}^{\varepsilon}(r)\right|^{2(p-1)}\left\|\partial_{z} \phi_{n, h}^{\varepsilon}(r)\right\|^{2} d r \\
& +C_{1} \int_{0}^{t \wedge \tau_{N}}\left|\partial_{z} \phi_{n, h}^{\varepsilon}(r)\right|^{2 p}\left\|\phi_{n, h}^{\varepsilon}(r)\right\|^{2} d r,
\end{aligned}
$$

and

$$
\begin{aligned}
J_{2}(t) & =2 p \int_{0}^{t \wedge \tau_{N}}\left|\left\langle\partial_{z z} u_{n, h}^{\varepsilon}, \int_{-h}^{z} \partial_{x} \theta_{n, h}^{\varepsilon} d z\right\rangle\right|\left|\partial_{z} \phi_{n, h}^{\varepsilon}(r)\right|^{2(p-1)} d r \\
& =2 p \int_{0}^{t \wedge \tau_{N}}\left|\left\langle\partial_{z} u_{n, h}^{\varepsilon}, \partial_{x} \theta_{n, h}^{\varepsilon}\right\rangle\right|\left|\partial_{z} \phi_{n, h}^{\varepsilon}(r)\right|^{2(p-1)} d r \\
& \leq 2 p \int_{0}^{t \wedge \tau_{N}}\left\|\theta_{n, h}^{\varepsilon}\right\|\left|\partial_{z} \phi_{n, h}^{\varepsilon}(r)\right|^{2 p-1} d r \\
& \leq \frac{1}{4} \sup _{0 \leq s \leq t \wedge \tau_{N}}\left|\partial_{z} \phi_{n, h}^{\varepsilon}(s)\right|^{2 p}+C_{2}\left(\int_{0}^{t \wedge \tau_{N}}\left\|\theta_{n, h}^{\varepsilon}(r)\right\|^{2} d r\right)^{2 p} .
\end{aligned}
$$

As we obtained the estimate (3.14), we also have

$$
\begin{aligned}
J_{3} \leq & \frac{1}{12} \int_{0}^{t \wedge \tau_{N}}\left|\partial_{z} \phi_{n, h}^{\varepsilon}(r)\right|^{2(p-1)}\left\|\partial_{z} \phi_{n, h}^{\varepsilon}(r)\right\|^{2} d r \\
& +\frac{1}{4} \sup _{0 \leq s \leq t \wedge \tau_{N}}\left|\partial_{z} \phi_{n, h}^{\varepsilon}\right|^{2 p}+C_{3}\left(\left|\partial_{z} f\right|_{L^{2}\left(0, t \wedge \tau_{N} ; H\right)}^{2 p}+\left|\partial_{z} q\right|_{L^{2}\left(0, t \wedge \tau_{N} ; H\right)}^{2 p}\right)
\end{aligned}
$$

As in (3.15), using the Cauchy-Schwarz inequality and (B.2), we get

$$
\begin{aligned}
J_{5} \leq & \frac{1}{12} \int_{0}^{t \wedge \tau_{N}}\left\|\partial_{z} \phi_{n, h}^{\varepsilon}(r)\right\|^{2}\left|\partial_{z} \phi_{n, h}^{\varepsilon}(r)\right|^{2(p-1)} d r+C_{5} \int_{0}^{t \wedge \tau_{N}}|h(r)|_{0}^{2}\left|\partial_{z} \phi_{n, h}^{\varepsilon}(r)\right|^{2 p} d r \\
& +\frac{1}{12} \int_{0}^{t \wedge \tau_{N}}\left|\partial_{z} \phi_{n, h}^{\varepsilon}(r)\right|^{2(p-1)} d r .
\end{aligned}
$$

Using again (B.1), we deduce that

$$
\begin{aligned}
J_{6}(t)+J_{7}(t) \leq & 2 p^{2} \bar{K} \varepsilon \int_{0}^{t \wedge \tau_{N}}\left\|\partial_{z} \phi_{n, h}^{\varepsilon}(r)\right\|^{2}\left|\partial_{z} \phi_{n, h}^{\varepsilon}(r)\right|^{2(p-1)} d r \\
& +2 p^{2} \bar{K} \varepsilon \int_{0}^{t \wedge \tau_{N}}\left|\partial_{z} \phi_{n, h}^{\varepsilon}(r)\right|^{2(p-1)} d r .
\end{aligned}
$$

Finally, the Burkholder-Davies-Gundy inequality, (B.1), and Schwarz's inequality yield that for $t \in[0, T]$ and $\delta_{4}>0$,

$$
\begin{aligned}
\mathbb{E}\left(\sup _{0 \leq s \leq t}\left|J_{4}(s)\right|\right) & \leq 6 p \sqrt{\varepsilon} \mathbb{E}\left\{\int_{0}^{t \wedge \tau_{N}}\left|\partial_{z} \phi_{n, h}^{\varepsilon}(r)\right|^{2(2 p-1)}\left|\partial_{z} \sigma_{n, h}\left(\phi_{n, h}^{\varepsilon}(r)\right) P_{n}\right|_{L_{Q}}^{2} d r\right\}^{\frac{1}{2}} \\
& \leq \delta_{4} \mathbb{E}\left(\sup _{0 \leq s \leq t \wedge \tau_{N}}\left|\partial_{z} \phi_{n, h}^{\varepsilon}(s)\right|^{2 p}\right)+\frac{9 p^{2} \bar{K} \varepsilon}{\delta_{4}} \mathbb{E} \int_{0}^{t \wedge \tau_{N}}\left|\partial_{z} \phi_{n, h}^{\varepsilon}(r)\right|^{2(p-1)} d r
\end{aligned}
$$




$$
+\frac{9 p^{2} \bar{K} \varepsilon}{\delta_{4}} \mathbb{E} \int_{0}^{t \wedge \tau_{N}}\left\|\partial_{z} \phi_{n, h}^{\varepsilon}(r)\right\|^{2}\left|\partial_{z} \phi_{n, h}^{\varepsilon}(r)\right|^{2(p-1)} d r
$$

Using similar steps to those in Proposition 3.1, due to (3.20)-(3.25) we get (3.18).

Due to Ladyzhenskaya's inequality for two-dimensional domains, we now have the following bound in $L^{4}(\mathcal{M})$.

Proposition 3.3. Let $h \in \mathcal{A}_{M}$ and $\xi \in L^{4}(\Omega, H)$, and let $\varepsilon_{2,0}$ be defined as in Proposition 3.1 with $p=2$. Then there exists a constant

$$
C_{2}:=C_{2}\left(K, \tilde{K}, T, M,|f|_{L^{4}\left(\Omega ; L^{2}(0, T ; H)\right)},|q|_{L^{4}\left(\Omega ; L^{2}(0, T ; H)\right)}\right)
$$

such that

$$
\sup _{n} \mathbb{E} \int_{0}^{T}\left|\phi_{n, h}^{\varepsilon}(s)\right|_{L^{4}}^{4} d s \leq C_{2}\left(1+\mathbb{E}|\xi|^{4}\right)
$$

The following result is a consequence of Itô's formula.

LEMMA 3.3. Let $\rho^{\prime}:[0, T] \times \Omega \rightarrow[0+\infty)$ be adapted such that for almost every $\omega$ $t \rightarrow \rho^{\prime}(t, \omega) \in L^{1}([0, T])$ and for $t \in[0, T]$, set $\rho(t)=\int_{0}^{t} \rho^{\prime}(s) d s$. For $i=1,2$, let $\sigma_{i}$ satisfy Assumptions $(A)$ and $(B), \bar{\sigma}_{i} \in C\left([0, T] \times H, L_{Q}^{2}\right)$, and let $\bar{\sigma}$ satisfy Assumptions $\tilde{A}$ and B. Let $E$ satisfy condition (3.6) and $h_{\varepsilon} \in \mathcal{A}_{M}$. Let $\phi_{i} \in L^{2}([0, T], V) \cap L^{\infty}([0, T], H)$ a.s. and be such that $\phi_{i}(0)=\xi \in L^{4}(\Omega, H)$, for $\xi \mathcal{F}_{0}$-measurable, and satisfy the equation

$$
d \phi_{i}(t)=R\left(\phi_{i}(t)\right) d t+\sqrt{\varepsilon} \sigma_{i}\left(t, \phi_{i}(t)\right) d W(t)+\left(\bar{\sigma}\left(t, \phi_{i}(t)\right)+\bar{\sigma}_{i}\left(t, \phi_{i}(t)\right)\right) h_{\varepsilon}(t) d t .
$$

Let $\Phi=\phi_{1}-\phi_{2}$. Then for every $t \in[0, T]$,

$$
\begin{aligned}
e^{-\rho(t)}|\Phi(t)|^{2} \leq & \int_{0}^{t} e^{-\rho(s)}\left\{-\frac{1}{2}\|\Phi(s)\|^{2}+\varepsilon\left|\sigma_{1}\left(s, \phi_{1}(s)\right)-\sigma_{2}\left(s, \phi_{2}(s)\right)\right|_{L_{Q}^{2}}^{2}\right. \\
& \left.+|\Phi(s)|^{2}\left[-\rho^{\prime}(s)+2 C\left(1+\left|\partial_{z} \phi_{2}(s)\right|^{4}\right)+C\left\|\phi_{2}(s)\right\|^{2}+C\left|h_{\varepsilon}(s)\right|_{0}^{2}\right]\right\} d s \\
& +2 \int_{0}^{t} e^{-\rho(s)}\left(\bar{\sigma}_{1}(s)-\bar{\sigma}_{2}(s), \Phi(s)\right) d s+I(t)
\end{aligned}
$$

where $I(t)=2 \sqrt{\varepsilon} \int_{0}^{t} e^{-\rho(s)}\left(\left[\sigma_{1}\left(s, \phi_{1}(s)\right)-\sigma_{2}\left(s, \phi_{2}(s)\right)\right] d W(s), \Phi(s)\right)$.

Proof. Itô's formula, (3.6), and condition ( $\tilde{A} .2)$ imply that for $t \in[0, T]$,

$$
\begin{aligned}
& \quad e^{-\rho(t)}|\Phi(t)|^{2} \\
& =\int_{0}^{t} e^{-\rho(s)}\left\{-\rho^{\prime}(s)|\Phi(s)|^{2}+\varepsilon\left|\sigma_{1}\left(s, \phi_{1}(s)\right)-\sigma_{2}\left(s, \phi_{2}(s)\right)\right|_{L_{Q}}^{2}\right. \\
& \left.\quad+2\left\langle E\left(\phi_{1}(s)\right)-E\left(\phi_{2}(s)\right), \Phi(s)\right\rangle+2\left(\bar{\sigma}\left(s, \phi_{1}(s)\right) h_{\varepsilon}(s)-\bar{\sigma}\left(s, \phi_{2}(s)\right) h_{\varepsilon}(s), \Phi(s)\right)\right\} d s \\
& \quad+\int_{0}^{t} e^{-\rho(s)} 2\left(\left[\bar{\sigma}_{1}(s)-\bar{\sigma}_{2}(s)\right] h_{\varepsilon}(s), \Phi(s)\right) d s+I(t) \\
& \leq \int_{0}^{t} e^{-\rho(s)}\left\{-\rho^{\prime}(s)|\Phi(s)|^{2}+\varepsilon\left|\sigma_{1}\left(s, \phi_{1}(s)\right)-\sigma_{2}\left(s, \phi_{2}(s)\right)\right|_{L_{Q}}^{2}-\|\Phi(s)\|^{2}\right. \\
& \left.\quad+2 C|\Phi(s)||| \Phi(s)\|\| \phi_{2}(s)\left\|+2 C\left(1+\left|\partial_{z} \phi_{2}(s)\right|^{4}\right)|\Phi(s)|^{2}+2 C \sqrt{\tilde{L}}\right\| \Phi(s) \|\left|h_{\varepsilon}(s)\right|_{0}|\Phi(s)|\right\} d s
\end{aligned}
$$




$$
+\int_{0}^{t} e^{-\rho(s)} 2\left(\bar{\sigma}_{1}(s)-\bar{\sigma}_{2}(s), \Phi(s)\right) d s+I(t) .
$$

The inequalities

$$
2 C|\Phi(s)|\|\Phi(s)\|\left\|\phi_{2}(s)\right\| \leq \frac{1}{4}\|\Phi(s)\|^{2}+C\left\|\phi_{2}(s)\right\|^{2}|\Phi(s)|^{2}
$$

and

$$
2 C \sqrt{\tilde{L}}\|\Phi(s)\|\left|h_{\varepsilon}(s)\right|_{0}|\Phi(s)| \leq \frac{1}{4}\|\Phi(s)\|^{2}+C\left|h_{\varepsilon}(s)\right|_{0}^{2}|\Phi(s)|^{2}
$$

conclude the proof of (3.28).

Proof of Theorem 3.1: As in [13], due to monotonicity property (3.6), a priori estimates (3.9), (3.18), and Lemma 3.3, we obtain Theorem 3.1.

\section{Large deviations}

Since the PEs are a large scale model, one may neglect the effect of small scale and intermediate scale in its modeling. One may consider this effect by adding small noise in the equations. Large deviations theory is concerned with the study of precise asymptotics governing the decay rate of probabilities of rare events. A classical area of large deviations is the Wentzell-Freidlin theory that deals with path probability asymptotics for small noise stochastic dynamical systems. More precisely, we will put a bound on the probability that the random perturbed trajectory goes very far from the unperturbed trajectory, and see the rate at which this probability goes to zero as the noise shrinks $(\varepsilon \rightarrow 0)$. We consider large deviations via a weak convergence approach (originated with Budhiraja, Dupuis [1, 2], and Sritharan and Sundar [42], Duan and Millet [13], among others). In this paper, the idea of the proof for large deviations is the same as [13], so here we only give the outline of the proof. Firstly, we recall some classical definitions with large deviations.

DEFINITION 4.1. The random family $\left\{\phi^{\varepsilon}\right\}$ is said to satisfy a large deviation principle on $X$ with the good rate function $I$ if the following conditions hold:

$I$ is a good rate function. The function function $I: X \rightarrow[0, \infty]$ is such that for each $M \in[0, \infty[$ the level set $\{\phi \in X: I(\phi) \leq M\}$ is a compact subset of $X$.

For $A \in \mathcal{B}(X)$, set $I(A)=\inf _{\phi \in A} I(\phi)$.

Large deviation upper bound. For each closed subset $F$ of $X$ :

$$
\limsup _{\varepsilon \rightarrow 0} \varepsilon \log \mathbb{P}\left(\phi^{\varepsilon} \in F\right) \leq-I(F) .
$$

Large deviation lower bound. For each open subset $G$ of $X$ :

$$
\liminf _{\varepsilon \rightarrow 0} \varepsilon \log \mathbb{P}\left(\phi^{\varepsilon} \in G\right) \geq-I(G) .
$$

To establish the large deviation principle, we need to strengthen the hypothesis on the growth condition and Lipschitz property of $\sigma$ (and $\tilde{\sigma}$ ) as follows:

There exist positive constants $K$ and $L$ such that

(A.4) $|\sigma(t, \phi)|_{L_{Q}}^{2} \leq K\left(1+|\phi|^{2}\right), \forall t \in[0, T], \forall \phi \in V$.

(A.5) $|\sigma(t, \phi)-\sigma(t, \psi)|_{L_{Q}}^{2} \leq L|\phi-\psi|^{2}, \forall t \in[0, T], \forall \phi, \psi \in V$.

The following theorem is the main result of this section. 
Theorem 4.1. Suppose $\sigma$ does not depend on time and satisfies (A.1), (B), (A.4), and (A.5), and let $\phi^{\varepsilon}$ be the solution of the stochastic Primitive Equations (2.23). Then $\left\{\phi^{\varepsilon}\right\}$ satisfies the large deviation principle in $C([0, T] ; H) \cap L^{2}((0, T) ; V)$, with the good rate function

$$
I_{\xi}(\psi)=\inf _{\left\{h \in L^{2}\left(0, T ; H_{0}\right): \psi=\mathcal{G}^{0}\left(\int_{0} h(s) d s\right)\right\}}\left\{\frac{1}{2} \int_{0}^{T}|h(s)|_{0}^{2} d s\right\} .
$$

Here the infimum of an empty set is taken as infinity.

The proof of the large deviation principle will use the following technical lemma which studies time increments of the solution to the stochastic control equation. For any integer $k=0, \cdots, 2^{n}-1$, and $s \in\left[k T 2^{-n},(k+1) T 2^{-n}\left[\right.\right.$, set $\underline{s}_{n}=k T 2^{-n}$ and $\bar{s}_{n}=$ $(k+1) T 2^{n}$. Given $N>0, h \in \mathcal{A}_{M}, \varepsilon \geq 0$ small enough, let $\phi_{h}^{\varepsilon} \underline{s}_{n}$ denote the solution to (2.29) given by Theorem 3.1, and for $t \in[0, T]$ let

$$
G_{N}(t)=\left\{\omega:\left(\sup _{0 \leq s \leq t}\left|\phi_{h}^{\varepsilon}(s)(\omega)\right|^{2}\right) \vee\left(\int_{0}^{t}\left\|\phi_{h}^{\varepsilon}(s)(\omega)\right\|^{2} d s\right) \vee\left(\sup _{0 \leq s \leq t}\left|\partial_{z} \phi_{h}^{\varepsilon}(s)(\omega)\right|^{2} \leq N\right)\right\} .
$$

Lemma 4.2. Let $M, N>0, \sigma$ and $\tilde{\sigma}$ satisfy the Assumptions (A.1), (B), (A.4), and (A.5), and $\partial_{z} \xi, \xi \in L^{4}(H)$. Then there exists a positive constant

$$
\begin{gathered}
C:=C\left(K, L,|f|_{L^{4}\left(\Omega ; L^{2}(0, T ; H)\right)},|q|_{L^{4}\left(\Omega ; L^{2}(0, T ; H)\right)},\left|\partial_{z} f\right|_{L^{4}\left(\Omega ; L^{2}(0, T ; H)\right)},\right. \\
\left.\left|\partial_{z} q\right|_{L^{4}\left(\Omega ; L^{2}(0, T ; H)\right)}, T, M, N, \varepsilon_{0}\right)
\end{gathered}
$$

such that for any $h \in \mathcal{A}_{M}$ and $\varepsilon \in\left[0, \varepsilon_{0}\right]$,

$$
I_{n}(h, \varepsilon):=\mathbb{E}\left[1_{G_{N}(T)} \int_{0}^{T}\left|\phi_{h}^{\varepsilon}(s)-\phi_{h}^{\varepsilon}\left(\bar{s}_{n}\right)\right|^{2} d s\right] \leq C 2^{-\frac{n}{2}} .
$$

Proof. The proof is close to that of Lemma 4.2 in [13], and we omit the details here.

Now we return to the setting of Theorem 4.1. Let $\varepsilon_{0}$ be defined as in Theorem 3.1 and $\left(h_{\varepsilon}, 0<\varepsilon \leq \varepsilon_{0}\right)$ be a family of random elements taking values in $\mathcal{A}_{M}$. Let $\phi_{h_{\varepsilon}}^{\varepsilon}$ be the solution of the corresponding stochastic control equation with initial condition $\phi_{h_{\varepsilon}}^{\varepsilon}(0)=\xi \in H$ :

$$
d \phi_{h_{\varepsilon}}^{\varepsilon}+\left[A \phi_{h_{\varepsilon}}^{\varepsilon}+B\left(\phi_{h_{\varepsilon}}^{\varepsilon}\right)+F\left(\phi_{h_{\varepsilon}}^{\varepsilon}\right)\right] d t=R d t+\sigma\left(\phi_{h_{\varepsilon}}^{\varepsilon}\right) h_{\varepsilon} d t+\sqrt{\varepsilon} \sigma\left(\phi_{h_{\varepsilon}}^{\varepsilon}\right) d W(t) .
$$

Note that $\phi_{h_{\varepsilon}}^{\varepsilon}=\mathcal{G}^{\varepsilon}\left(\sqrt{\varepsilon}\left(W .+\frac{1}{\sqrt{\varepsilon}} \int_{0}^{\cdot} h_{\varepsilon}(s) d s\right)\right)$ due to the uniqueness of the solution.

For all $\omega$ and $h \in L^{2}\left([0, T], H_{0}\right)$, let $\phi_{h}$ be the solution of the corresponding control equation with initial condition $\phi_{h}(0)=\xi(\omega)$ :

$$
d \phi_{h}+\left[A \phi_{h}+B\left(\phi_{h}\right)+F\left(\phi_{h}\right)\right] d t=R d t+\sigma\left(\phi_{h}\right) h d t .
$$

Note that here we may assume that $h$ and $\xi$ are random, but $\phi_{h}$ may defined pointwise by (4.6).

Let $\mathcal{C}_{0}=\left\{\int_{0}^{\cdot} h(s) d s: h \in L^{2}\left([0, T], H_{0}\right)\right\} \subset C\left([0, T], H_{0}\right)$. For every $\omega \in \Omega$, define $\mathcal{G}^{0}$ : $C\left([0, T], H_{0}\right) \rightarrow X$ by $\mathcal{G}^{0}(g)(\omega)=\phi_{h}(\omega)$ for $g=\int_{0}^{\cdot} h(s) d s \in \mathcal{C}_{0}$ and $\mathcal{G}^{0}(g)=0$ otherwise. 
Proposition 4.2 (Weak convergence). Suppose that $\sigma$ does not depend on time and satisfies the Assumptions (A.1), (B), (A.4), and (A.5). Let $\xi \in Y$ be $\mathcal{F}_{0}$ measurable such that $E|\xi|_{H}^{4}<+\infty, E\left|\partial_{z} \xi\right|_{H}^{4}<+\infty$, and let $h_{\varepsilon}$ converge to $h$ in distribution as random elements taking values in $\mathcal{A}_{M}$. (Note that here $\mathcal{A}_{M}$ is endowed with the weak topology induced by the norm (3.1)). Then as $\varepsilon \rightarrow 0, \phi_{h_{\varepsilon}}^{\varepsilon}$ converges in distribution to $\phi_{h}$ in $X=C([0, T] ; H) \cap L^{2}((0, T) ; V)$ endowed with the norm (3.1). That is, $\mathcal{G}^{\varepsilon}\left(\sqrt{\varepsilon}\left(W .+\frac{1}{\sqrt{\varepsilon}} \int_{0}^{\cdot} h_{\varepsilon}(s) d s\right)\right)$ converges in distribution to $\mathcal{G}^{0}\left(\int_{0}^{\cdot} h(s) d s\right)$ in $X$, as $\varepsilon \rightarrow 0$.

The proof can be obtained by delicate estimates and the method of [13].

The following compactness result is the second ingredient which allows us to transfer the LDP from $\sqrt{\varepsilon} W$ to $u^{\varepsilon}$. Its proof is similar to that of Proposition 4.2, so we refer to [13].

Proposition 4.3 (Compactness). Let $M$ be any fixed finite positive number and let $\xi \in Y$ be deterministic. Define

$$
K_{M}=\left\{\phi_{h} \in C([0, T] ; H) \cap L^{2}((0, T) ; V): h \in S_{M}\right\},
$$

where $\phi_{h}$ is the unique solution of the deterministic control equation

$$
\begin{aligned}
& d \phi_{h}(t)+\left[A \phi_{h}(t)+B\left(\phi_{h}(t)\right)+R \phi_{h}(t)\right] d t=F \phi_{h}(t) d t+\sigma\left(\phi_{h}(t)\right) h(t) d t, \\
& \phi_{h}(0)=\xi
\end{aligned}
$$

and $\sigma$ does not depend on time and satisfies (A.1), (B), (A.4), and (A.5). Then $K_{M}$ is a compact subset of $X$.

Proof of Theorem 4.1: Propositions 4.3 and 4.2 imply that $\left\{\phi^{\varepsilon}\right\}$ satisfies the Laplace principle, which is equivalent to the large deviation principle in $X=$ $C([0, T], H) \cap L^{2}((0, T), V)$ with the above-mentioned rate function; see Theorem 4.4 in [1] or Theorem 5 in [2].

Acknowledgment.The authors are indebted to the referee for giving some important suggestions which improved the presentations of this paper.

\section{REFERENCES}

[1] A. Budhiraja and P. Dupuis, A variational representation for positive functionals of infinite dimensional Brownian motion, Prob. and Math. Stat., 20, 39-61, 2000.

[2] A. Budhiraja, P. Dupuis and V. Maroulas, Large deviations for infinite dimensional stochastic dynamical systems, Ann. Prob., 36, 1390-1420, 2008.

[3] C. Cao and E.S. Titi, Global well-posedness of the three-dimensional primitive equations of large scale ocean and atmosphere dynamics, Ann. Math., 166, 245-267, 2007.

[4] S. Cerrai and M. Rockner, Large deviations for stochastic reaction-diffusion systems with multiplicative noise and non-Lipschitz reaction term, Ann. Prob., 32, 1100-1139, 2004.

[5] M.H. Chang, Large deviations for the Navier-Stokes equations with small stochastic perturbations, Appl. Math. Comput., 76, 65-93, 1996.

[6] F. Chenal and A. Millet, Uniform large deviations for parabolic SPDEs and applications, Stochastic Process. Appl., 72, 161-186, 1997.

[7] P.L. Chow, Large deviation problem for some parabolic Itô equations, Commun. Pure Appl. Math., 45, 97-120, 1992.

[8] I. Chueshov and A. Millet, Stochastic 2D hydrodynamical type systems: Well posedness and large deviations, Appl. Math. Optim., 61, 379-420, 2010.

[9] B. Cushman-Roisin and J.M. Beckers, Introduction to Geophysical Fluid Dynamics: Physical and Numerical Aspects, Academic Press, 2007. 
[10] G. Da Prato and J. Zabczyk, Stochastic Equations in Infinite Dimensions, Cambridge University Press, 1992.

[11] A. Debussche, N. Glatt-Holtz, and R. Temam, Local martingale and pathwise solutions for an abstract fluids model, Physica D, 240, 1123-1144, 2011.

[12] A. Du, J. Duan, and H. Gao, Small probability events for two-layer geophysical flows under uncertainty, arXiv:0810.2818, October, 2008.

[13] J. Duan and A. Millet, Large deviations for the Boussinesq equations under random influences, Stochastic Proc. Appl., 119, 2052-2081, 2009.

[14] B. Ewald, M. Petcu, and R. Temam, Stochastic solutions of the two-dimensional primitive equations of the ocean and atmosphere with an additive noise, Anal. Appl., 5, 183-198, 2007.

[15] F. Flandoli, An introduction to 3D stochastic fluid dynamics, SPDE in Hydrodynamic: Recent Progress and Prospects, Lecture Notes in Math., 1942, Springer, Berlin, 51-150, 2008.

[16] C. Frankignoul and K. Hasselmann, Stochastic climate models, Part II: Application to seasurface temperature anomalies and thermocline variability, Tellus, 29, 289-305, 1977.

[17] M.I. Freidlin and A.D. Wentzell, Reaction-diffusion equation with randomly perturbed boundary condition, Annals. Prob., 20(2), 963-986, 1992.

[18] H. Gao and C. Sun, Random attractor for the $3 D$ viscous stochastic primitive equations with additive noise, Stoch. Dyn., 9, 293-313, 2009.

[19] N. Glatt-Holtz and R. Temam, Pathwise solutions of the 2-d stochastic primitive equations, Appl. Math. Opt., 63, 401-433, 2011.

[20] N. Glatt-Holtz and M. Ziane, Strong pathwise solutions of the stochastic Navier-Stokes system, Adv. Diff. Equ., 14, 567-600, 2009.

[21] N. Glatt-Holtz and M. Ziane, The stochastic primitive equations in two space dimensions with multiplicative noise, Disc. Cont. Dyn. Sys. Series B, 10(4), 801-822, 2008.

[22] F. Guillén-Gonzáez, N. Masmoudi, and M.A. Rodríguez-Bellido, Anisotropic estimates and strong solutions of the Primitive Equations, Diff. Integral Eqs., 14, 1381-1408, 2001.

[23] B. Guo and D. Huang, 3D stochastic primitive equations of the large-scale ocean: Global wellposedness and attractors, Commun. Math. Phys., 286(2), 697-723, 2009.

[24] C. Hu, R. Temam, and M. Ziane, The primitive equations on the large scale ocean under the small depth hypothesis, Discrete Contin. Dyn. Syst., 9, 97-131, 2003.

[25] N. Ju, The global attractor for the solutions to the $3 d$ viscous primitive equations, Disc. Contin. Dyn. Syst., 17, 159-179, 2007.

[26] G. Kallianpur and J. Xiong, Large deviations for a class of stochastic partial differential equations, Ann. Prob., 24, 320-345, 1996.

[27] G. Kobelkov, Existence of a solution in "whole" for the large-scale ocean dynamics equations, C.R. Math. Acad. Sci. Paris, 343, 283-286, 2006.

[28] G.M. Kobelkov and V.B. Zalesny, Existence and uniqueness of a solution to primitive equations with stratification 'in the large', Russian J. Numer. Anal. Math. Modelling, 23, 39-61, 2008.

[29] I. Kukavica and M. Ziane, On the regularity of the primitive equations of the ocean, Nonlin., 20, 2739-2753, 2007.

[30] J.L. Lions, R. Temam, and S. Wang, Models for the coupled atmosphere and ocean, Comput. Mech. Adv., 1, 1-120, 1993.

[31] J.L. Lions, R. Temam, and S. Wang, New formulations of the primitive equations of atmosphere and applications, Nonlin., 5, 237-288, 1992.

[32] J.L. Lions, R. Temam, and S. Wang, On the equations of the large-scale ocean, Nonlin., 5, 1007-1053, 1992.

[33] R. Mikulevicius and B.L. Rozovskii, Global L $L^{2}$-solutions of stochastic Navier-Stokes equations, Ann. Probab., 33, 137-176, 2005.

[34] P. Müller, Stochastic Forcing of Quasi-Geostrophic Eddies, Stochastic Modelling in Physical Oceanography, edited by R. J. Adler, P. Müller, B. Rozovskii, Basel: Birkhaüser, 1996.

[35] J. Pedlosky, Geophysical Fluid Dynamics, Springer-Verlag, New York, 1987.

[36] S. Peszat, Large deviation estimates for stochastic evolution equations, Prob. Theory Rel. Fields, 98, 113-136, 1994.

[37] M. Petcu, Gevrey class regularity for the primitive equations in space dimension 2, Asymptot. Anal., 39, 1-13, 2004.

[38] M. Petcu, R. Temam, and D. Wirosoetisno, Existence and regularity results for the primitive equations in two space dimensions, Commun. Pure Appl. Anal., 3, 115-131, 2004.

[39] M. Petcu, R. Temam, and M. Ziane, Some mathematical problems in geophysical fluid dynam$i c s$, in Special Volume on Computational Methods for the Atmosphere and the Oceans, Handbook of Numerical Analysis, Elsevier, 14, 577-750, 2008.

[40] O.M. Phillips, On the generation of waves by turbulent winds, J. Fluid. Mech., 2, 417-445, 
1957.

[41] R. Sowers, Large deviations for a reaction-diffusion system with non-Gaussian perturbations, Ann. Prob., 20, 504-537, 1992.

[42] S.S. Sritharan and P. Sundar, Large deviations for the two-dimensional Navier-Stokes equations with multiplicative noise, Stoch. Proc. and Appl., 116, 1636-1659, 2006.

[43] R. Temam and M. Ziane, Some Mathematical Problems in Geophysical Fluid Dynamics, Handbook of Mathematical Fluid Dynamics, 3, 2004.

[44] J. Zabczyk, On Large Deviations for Stochastic Evolution Equations. Stochastic Systems and Optimization, Lecture Notes on Control and Inform. Sci., Springer, Berlin, 1988. 